\title{
ERRATUM
}

\section{Erratum to: Diversity and regeneration status of woody species in Tara Gedam and Abebaye forests, northwestern Ethiopia}

Haileab Zegeye • Demel Teketay • Ensermu Kelbessa

(C) Northeast Forestry University and Springer-Verlag Berlin Heidelberg 2013

Erratum to: Journal of Forestry Research (2011) 22(3): 315-328

DOI 10.1007/s11676-011-0176-6

The original version of this article unfortunately contained one mistake in the column (IVI) of Tables 2 and 3 due to wrong formula. The corrected Table 2 and Table 3 should be:

Table 2. Density, relative density, frequency, relative frequency, basal area, relative basal area and IVI of woody species in Tara Gedam forest

\begin{tabular}{|c|c|c|c|c|c|c|c|}
\hline Species & Density & $\begin{array}{c}\text { Relative } \\
\text { density (\%) }\end{array}$ & $\begin{array}{c}\text { Frequency } \\
(\%)\end{array}$ & $\begin{array}{c}\text { Relative } \\
\text { frequency }(\%)\end{array}$ & $\begin{array}{c}\text { Basal area } \\
\left(\mathrm{m}^{2} \cdot \mathrm{ha}^{-1}\right)\end{array}$ & $\begin{array}{c}\text { Relative basal } \\
\text { area }(\%)\end{array}$ & IVI \\
\hline Acacia abyssinica & 29.76 & 0.99 & 19.05 & 1.07 & 2.79 & 2.41 & 4.47 \\
\hline Acacia lahai & 21.43 & 0.71 & 9.52 & 0.53 & 0.53 & 0.46 & 1.7 \\
\hline Acacia pilispina & 60.71 & 2.02 & 23.81 & 1.34 & 0.19 & 0.16 & 3.52 \\
\hline Acanthus polystachius & 55.95 & 1.86 & 28.57 & 1.60 & 0.04 & 0.03 & 3.49 \\
\hline Acokanthera schimperi & 33.33 & 1.11 & 9.52 & 0.53 & - & - & - \\
\hline Albizia gummifera & 252.38 & 8.41 & 80.95 & 4.55 & 10.58 & 9.17 & 22.13 \\
\hline Apodytes dimidiata & 20.24 & 0.67 & 14.29 & 0.80 & 3.39 & 2.94 & 4.41 \\
\hline Barleria ventricosa & 3.57 & 0.12 & 4.76 & 0.27 & - & - & - \\
\hline Bersama abyssinica & 33.33 & 1.11 & 23.81 & 1.34 & 0.44 & 0.38 & 2.83 \\
\hline Bridelia micrantha & 5.95 & 0.20 & 9.52 & 0.53 & 1.39 & 1.21 & 1.94 \\
\hline Brucea antidysenterica & 19.05 & 0.63 & 14.29 & 0.80 & 0.01 & 0.01 & 1.44 \\
\hline
\end{tabular}

The online version of the original article can be found at: http://dx.doi.org/10.1007/s11676-011-0176-6

\section{Haileab Zegeye}

Department of Biology, Debre Markos University, P.O. Box 269, Debre

Markos, Ethiopia.

E-mail: haileabzegeye@,fastmail.fm, haileabzt@yahoo.com

Demel Teketay

University of Botswana, Okavango Research Institute, Shorobe Road,

Private Bag 285, Maun, Botswana.

E-mail: demelteketay.fanta@orc.ub.bw, dteketay@yahoo.com

Ensermu Kelbessa

The National Herbarium, Addis Ababa University, P.O. Box 3434,

Addis Ababa, Ethiopia.

E-mail: ensermuk2002@yahoo.co.uk, ensermu@bio.aau.edu.et

Corresponding editor: Yu Lei 


\begin{tabular}{|c|c|c|c|c|c|c|c|}
\hline \multicolumn{8}{|l|}{ Continued Table 2} \\
\hline Species & Density & $\begin{array}{c}\text { Relative } \\
\text { density }(\%)\end{array}$ & $\begin{array}{c}\text { Frequency } \\
(\%)\end{array}$ & $\begin{array}{c}\text { Relative } \\
\text { frequency }(\%)\end{array}$ & $\begin{array}{c}\text { Basal area } \\
\left(\mathrm{m}^{2} \cdot \mathrm{ha}^{-1}\right)\end{array}$ & $\begin{array}{c}\text { Relative basal } \\
\text { area (\%) }\end{array}$ & IVI \\
\hline Buddleja polystachya & 15.48 & 0.52 & 14.29 & 0.80 & 0.01 & 0.01 & 1.33 \\
\hline Calotropis procera & 4.76 & 0.16 & 4.76 & 0.27 & - & - & - \\
\hline Calpurnia aurea & 245.24 & 8.17 & 61.90 & 3.48 & 1.08 & 0.94 & 12.59 \\
\hline Capparis tomentosa & 30.95 & 1.03 & 33.33 & 1.87 & - & - & - \\
\hline Carissa spinarum & 96.43 & 3.21 & 71.43 & 4.01 & 0.58 & 0.50 & 7.72 \\
\hline Cissus quadrangularis & 2.38 & 0.08 & 4.76 & 0.27 & - & - & - \\
\hline Clausena anisata & 23.81 & 0.79 & 23.81 & 1.34 & 0.02 & 0.02 & 2.15 \\
\hline Clematis longicauda & 4.76 & 0.16 & 9.52 & 0.53 & - & - & - \\
\hline Clerodendrum myricoides & 2.38 & 0.08 & 4.76 & 0.27 & 0.01 & 0.01 & 0.36 \\
\hline Clutia abyssinica & 2.38 & 0.08 & 4.76 & 0.27 & - & - & - \\
\hline Clutia lanceolata & 59.52 & 1.98 & 28.57 & 1.60 & 0.03 & 0.03 & 3.61 \\
\hline Combretum molle & 11.90 & 0.40 & 19.05 & 1.07 & 0.79 & 0.68 & 2.15 \\
\hline Cordia africana & 13.10 & 0.44 & 19.05 & 1.07 & 0.40 & 0.35 & 1.86 \\
\hline Croton macrostachyus & 61.90 & 2.06 & 47.62 & 2.67 & 0.47 & 0.41 & 5.14 \\
\hline Dodonaea angustifolia & 154.76 & 5.16 & 33.33 & 1.87 & 0.25 & 0.22 & 7.25 \\
\hline Dombeya torrida & 9.52 & 0.32 & 4.76 & 0.27 & 0.39 & 0.34 & 0.93 \\
\hline Dovyalis abyssinica & 8.33 & 0.28 & 14.29 & 0.80 & 0.37 & 0.32 & 1.4 \\
\hline Ehretia cymosa & 3.57 & 0.12 & 4.76 & 0.27 & - & - & - \\
\hline Ekebergia capensis & 107.14 & 3.57 & 38.10 & 2.14 & 9.36 & 8.11 & 13.82 \\
\hline Embelia schimperi & 2.38 & 0.08 & 4.76 & 0.27 & - & - & - \\
\hline Euclea racemosa subsp. schimperi & 7.14 & 0.24 & 9.52 & 0.53 & 0.09 & 0.08 & 0.85 \\
\hline Euphorbia abyssinica & 20.24 & 0.67 & 14.29 & 0.80 & 0.12 & 0.11 & 1.58 \\
\hline Ficus sur & 7.14 & 0.24 & 9.52 & 0.53 & 1.20 & 1.04 & 1.81 \\
\hline Ficus sycomorus & 3.57 & 0.12 & 4.76 & 0.27 & 0.03 & 0.02 & 0.41 \\
\hline Ficus thonningii & 2.38 & 0.08 & 4.76 & 0.27 & 0.06 & 0.05 & 0.4 \\
\hline Ficus vasta & 5.95 & 0.20 & 9.52 & 0.53 & 7.71 & 6.68 & 7.41 \\
\hline Gnidia glauca & 11.90 & 0.40 & 14.29 & 0.80 & - & - & - \\
\hline Grewia ferruginea & 41.67 & 1.39 & 42.86 & 2.41 & 0.23 & 0.20 & 4 \\
\hline Gymnema sylvestre & 2.38 & 0.08 & 4.76 & 0.27 & - & - & - \\
\hline Hymenodictyon floribundum & 2.38 & 0.08 & 4.76 & 0.27 & 0.26 & 0.23 & 0.58 \\
\hline Hypericum quartinianum & 29.76 & 0.99 & 23.81 & 1.34 & - & - & - \\
\hline Jasminum grandiflorum subsp. floribundum & 38.10 & 1.27 & 38.10 & 2.14 & - & - & - \\
\hline Juniperus procera & 21.43 & 0.71 & 14.29 & 0.80 & 0.11 & 0.10 & 1.61 \\
\hline Justicia schimperiana & 83.33 & 2.78 & 9.52 & 0.53 & - & - & - \\
\hline Lannea schimperi & 2.38 & 0.08 & 4.76 & 0.27 & 0.26 & 0.23 & 0.58 \\
\hline Lepidotrichilia volkensii & 4.76 & 0.16 & 4.76 & 0.27 & - & - & - \\
\hline Lippia adoensis & 22.62 & 0.75 & 19.05 & 1.07 & - & - & - \\
\hline Maesa lanceolata & 4.76 & 0.16 & 4.76 & 0.27 & 0.13 & 0.11 & 0.54 \\
\hline Maytenus arbutifolia & 45.24 & 1.51 & 23.81 & 1.34 & 0.07 & 0.06 & 2.91 \\
\hline Maytenus obscura & 4.76 & 0.16 & 9.52 & 0.53 & 0.58 & 0.50 & 1.19 \\
\hline Maytenus serrata & 60.71 & 2.02 & 38.10 & 2.14 & 0.01 & 0.01 & 4.17 \\
\hline Millettia ferruginea & 7.14 & 0.24 & 9.52 & 0.53 & 0.29 & 0.26 & 1.03 \\
\hline Mimusops kummel & 17.86 & 0.60 & 9.52 & 0.53 & 0.52 & 0.45 & 1.58 \\
\hline Myrsine africana & 9.52 & 0.32 & 4.76 & 0.27 & 0.03 & 0.03 & 0.62 \\
\hline Nuxia congesta & 41.67 & 1.39 & 33.33 & 1.87 & 1.60 & 1.39 & 4.65 \\
\hline Ocimum urticifolium & 53.57 & 1.79 & 19.05 & 1.07 & - & - & - \\
\hline Olea europaea subsp. cuspidata & 203.57 & 6.78 & 61.90 & 3.48 & 47.06 & 40.79 & 51.05 \\
\hline Opuntia ficus-indica & 4.76 & 0.16 & 4.76 & 0.27 & - & - & - \\
\hline Osyris quadripartita & 48.81 & 1.63 & 42.86 & 2.41 & 0.07 & 0.06 & 4.1 \\
\hline Otostegia integrifolia & 22.62 & 0.75 & 14.29 & 0.80 & - & - & - \\
\hline Pavetta abyssinica & 8.33 & 0.28 & 9.52 & 0.53 & 0.03 & 0.02 & 0.83 \\
\hline Pavetta oliveriana & 3.57 & 0.12 & 4.76 & 0.27 & - & - & - \\
\hline Pavonia urens & 4.76 & 0.16 & 4.76 & 0.27 & - & - & - \\
\hline
\end{tabular}




\begin{tabular}{|c|c|c|c|c|c|c|c|}
\hline \multicolumn{8}{|l|}{ Continued Table 2} \\
\hline Species & Density & $\begin{array}{c}\text { Relative } \\
\text { density (\%) }\end{array}$ & $\begin{array}{c}\text { Frequency } \\
(\%)\end{array}$ & $\begin{array}{c}\text { Relative } \\
\text { frequency }(\%)\end{array}$ & $\begin{array}{l}\text { Basal area } \\
\left(\mathrm{m}^{2} \cdot \mathrm{ha}^{-1}\right)\end{array}$ & $\begin{array}{c}\text { Relative basal } \\
\text { area }(\%)\end{array}$ & IVI \\
\hline Phoenix reclinata & 1.19 & 0.04 & 4.76 & 0.27 & - & - & - \\
\hline Phytolacca dodecandra & 5.95 & 0.20 & 4.76 & 0.27 & - & - & - \\
\hline Premna schimperi & 39.29 & 1.31 & 33.33 & 1.87 & 0.26 & 0.22 & 3.4 \\
\hline Protea gaguedi & 3.57 & 0.12 & 4.76 & 0.27 & 0.01 & 0.01 & 0.4 \\
\hline Prunus africana & 5.95 & 0.20 & 9.52 & 0.53 & 1.42 & 1.23 & 1.96 \\
\hline Pterolobium stellatum & 67.86 & 2.26 & 47.62 & 2.67 & - & - & - \\
\hline Rhamnus staddo & 39.29 & 1.31 & 33.33 & 1.87 & 0.09 & 0.08 & 3.26 \\
\hline Rhus glutinosa & 83.33 & 2.78 & 47.62 & 2.67 & 1.37 & 1.19 & 6.64 \\
\hline Rhus vulgaris & 29.76 & 0.99 & 14.29 & 0.80 & 0.02 & 0.02 & 1.81 \\
\hline Ritchiea albersii & 4.76 & 0.16 & 9.52 & 0.53 & 0.32 & 0.28 & 0.97 \\
\hline Rosa abyssinica & 15.48 & 0.52 & 23.81 & 1.34 & 0.02 & 0.02 & 1.88 \\
\hline Rubus steudneri & 2.38 & 0.08 & 4.76 & 0.27 & - & - & - \\
\hline Rumex nervosus & 42.86 & 1.43 & 23.81 & 1.34 & - & - & - \\
\hline Sapium ellipticum & 3.57 & 0.12 & 4.76 & 0.27 & 0.81 & 0.70 & 1.09 \\
\hline Schefflera abyssinica & 21.43 & 0.71 & 19.05 & 1.07 & 11.83 & 10.26 & 12.04 \\
\hline Schrebera alata & 89.29 & 2.98 & 57.14 & 3.21 & 2.85 & 2.47 & 8.66 \\
\hline Scolopia theifolia & 61.90 & 2.06 & 33.33 & 1.87 & 1.62 & 1.41 & 5.34 \\
\hline Senna didymobotrya & 17.86 & 0.60 & 4.76 & 0.27 & 0.03 & 0.03 & 0.9 \\
\hline Solanum anguivi & 4.76 & 0.16 & 9.52 & 0.53 & - & - & - \\
\hline Solanum giganteum & 23.81 & 0.79 & 4.76 & 0.27 & 0.12 & 0.10 & 1.16 \\
\hline Solanum incanum & 3.57 & 0.12 & 4.76 & 0.27 & - & - & - \\
\hline Solanum marginatum & 7.14 & 0.24 & 4.76 & 0.27 & - & - & - \\
\hline Steganotaenia araliacea & 8.33 & 0.28 & 14.29 & 0.80 & 0.07 & 0.06 & 1.14 \\
\hline Stereospermum kunthianum & 14.29 & 0.48 & 23.81 & 1.34 & 0.14 & 0.12 & 1.94 \\
\hline Syzygium guineense & 2.38 & 0.08 & 4.76 & 0.27 & 0.15 & 0.13 & 0.48 \\
\hline Teclea nobilis & 22.62 & 0.75 & 23.81 & 1.34 & 0.30 & 0.26 & 2.35 \\
\hline Urera hypselodendron & 2.38 & 0.08 & 4.76 & 0.27 & - & - & - \\
\hline Vernonia amygdalina & 19.05 & 0.63 & 14.29 & 0.80 & 0.01 & 0.01 & 1.44 \\
\hline Vernonia brachycalyx & 2.38 & 0.08 & 4.76 & 0.27 & - & - & - \\
\hline Vernonia congolensis & 3.57 & 0.12 & 4.76 & 0.27 & - & - & - \\
\hline Vernonia myriantha & 98.81 & 3.29 & 57.14 & 3.21 & 0.29 & 0.26 & 6.76 \\
\hline Ximenia americana & 7.14 & 0.24 & 9.52 & 0.53 & 0.01 & 0.01 & 0.78 \\
\hline Total & $3,001.19$ & 100.00 & $1,780.95$ & 100.00 & 115.36 & 100.00 & 300.00 \\
\hline
\end{tabular}

Table 3. Density, relative density, frequency, relative frequency, basal area, relative basal area and IVI of woody species in Abebaye forest

\begin{tabular}{|c|c|c|c|c|c|c|c|}
\hline Species & Density & $\begin{array}{c}\text { Relative } \\
\text { density (\%) }\end{array}$ & $\begin{array}{c}\text { Frequeny } \\
(\%)\end{array}$ & $\begin{array}{c}\text { Relative } \\
\text { frequency }(\%)\end{array}$ & $\begin{array}{c}\text { Basal area } \\
\left(\mathrm{m}^{2} \mathrm{ha}^{-1}\right)\end{array}$ & $\begin{array}{c}\text { Relative basal } \\
\text { area }(\%)\end{array}$ & IVI \\
\hline Acacia lahai & 486.11 & 17.06 & 77.78 & 3.63 & 8.01 & 16.20 & 36.89 \\
\hline Acacia seyal & 13.89 & 0.49 & 11.11 & 0.52 & - & - & - \\
\hline Acanthus polystachius & 261.11 & 9.16 & 88.89 & 4.15 & - & - & - \\
\hline Acokanthera schimperi & 13.89 & 0.49 & 22.22 & 1.04 & - & - & - \\
\hline Asparagus africanus & 5.56 & 0.19 & 11.11 & 0.52 & - & - & - \\
\hline Bersama abyssinica & 50.00 & 1.75 & 22.22 & 1.04 & 0.07 & 0.14 & 2.93 \\
\hline Bridelia micrantha & 5.56 & 0.19 & 11.11 & 0.52 & 0.02 & 0.03 & 0.74 \\
\hline Brucea antidysenterica & 5.56 & 0.19 & 11.11 & 0.52 & - & - & - \\
\hline Calpurnia aurea & 50.00 & 1.75 & 44.44 & 2.07 & 0.81 & 1.64 & 5.46 \\
\hline Capparis tomentosa & 102.78 & 3.61 & 100.00 & 4.66 & - & - & - \\
\hline Carissa spinarum & 94.44 & 3.31 & 77.78 & 3.63 & 0.41 & 0.82 & 7.76 \\
\hline Cissus quadrangularis & 41.67 & 1.46 & 33.33 & 1.55 & - & - & - \\
\hline Clematis hirsuta & 16.67 & 0.58 & 33.33 & 1.55 & - & - & - \\
\hline
\end{tabular}




\begin{tabular}{|c|c|c|c|c|c|c|c|}
\hline \multicolumn{8}{|l|}{ Continued Table 3} \\
\hline Species & Density & $\begin{array}{c}\text { Relative } \\
\text { density (\%) }\end{array}$ & $\begin{array}{c}\text { Frequeny } \\
(\%)\end{array}$ & $\begin{array}{c}\text { Relative } \\
\text { frequency }(\%)\end{array}$ & $\begin{array}{c}\text { Basal area } \\
\left(\mathrm{m}^{2} \mathrm{ha}^{-1}\right)\end{array}$ & $\begin{array}{c}\text { Relative basal } \\
\text { area (\%) }\end{array}$ & IVI \\
\hline Clerodendrum myricoides & 2.78 & 0.10 & 11.11 & 0.52 & - & - & - \\
\hline Combretum molle & 19.44 & 0.68 & 22.22 & 1.04 & 0.79 & 1.60 & 3.32 \\
\hline Commiphora habessinica & 8.33 & 0.29 & 11.11 & 0.52 & - & - & - \\
\hline Cordia africana & 16.67 & 0.58 & 44.44 & 2.07 & 0.88 & 1.77 & 4.42 \\
\hline Croton macrostachyus & 63.89 & 2.24 & 100.00 & 4.66 & 4.40 & 8.90 & 15.8 \\
\hline Dichrostachys cinerea & 11.11 & 0.39 & 22.22 & 1.04 & - & - & - \\
\hline Dodonaea angustifolia & 58.33 & 2.05 & 44.44 & 2.07 & - & - & - \\
\hline Dombeya torrida & 5.56 & 0.19 & 11.11 & 0.52 & 0.01 & 0.01 & 0.72 \\
\hline Dovyalis abyssinica & 13.89 & 0.49 & 22.22 & 1.04 & - & - & - \\
\hline Entada abyssinica & 22.22 & 0.78 & 11.11 & 0.52 & 0.52 & 1.04 & 2.34 \\
\hline Erythrina abyssinica & 8.33 & 0.29 & 22.22 & 1.04 & 0.01 & 0.03 & 1.36 \\
\hline Euclea racemosa subsp. schimperi & 27.78 & 0.97 & 44.44 & 2.07 & 0.04 & 0.09 & 3.13 \\
\hline Euphorbia tirucalli & 8.33 & 0.29 & 11.11 & 0.52 & 0.05 & 0.10 & 0.91 \\
\hline Ficus sur & 5.56 & 0.19 & 22.22 & 1.04 & 2.46 & 4.98 & 6.21 \\
\hline Ficus sycomorus & 2.78 & 0.10 & 11.11 & 0.52 & 2.18 & 4.41 & 5.03 \\
\hline Ficus vasta & 5.56 & 0.19 & 22.22 & 1.04 & 16.77 & 33.91 & 35.14 \\
\hline Flueggea virosa & 5.56 & 0.19 & 11.11 & 0.52 & - & - & - \\
\hline Gardenia ternifolia & 22.22 & 0.78 & 44.44 & 2.07 & - & - & - \\
\hline Grewia ferruginea & 27.78 & 0.97 & 33.33 & 1.55 & 0.04 & 0.09 & 2.61 \\
\hline Hibiscus macranthus & 19.44 & 0.68 & 22.22 & 1.04 & - & - & - \\
\hline Hypericum quartinianum & 8.33 & 0.29 & 22.22 & 1.04 & - & - & - \\
\hline Indigofera tinctoria & 5.56 & 0.19 & 11.11 & 0.52 & - & - & - \\
\hline Jasminum grandiflorum subsp. floribundum & 33.33 & 1.17 & 77.78 & 3.63 & - & - & - \\
\hline Lannea schimperi & 2.78 & 0.10 & 11.11 & 0.52 & 1.07 & 2.16 & 2.78 \\
\hline Leonotis ocymifolia & 16.67 & 0.58 & 22.22 & 1.04 & - & - & - \\
\hline Maesa lanceolata & 5.56 & 0.19 & 11.11 & 0.52 & 0.02 & 0.04 & 0.75 \\
\hline Maytenus serrata & 252.78 & 8.87 & 88.89 & 4.15 & - & - & - \\
\hline Nuxia congesta & 13.89 & 0.49 & 22.22 & 1.04 & 0.17 & 0.34 & 1.87 \\
\hline Ocimum urticifolium & 77.78 & 2.73 & 55.56 & 2.59 & - & - & - \\
\hline Opuntia ficus-indica & 11.11 & 0.39 & 11.11 & 0.52 & - & - & - \\
\hline Osyris quadripartita & 22.22 & 0.78 & 44.44 & 2.07 & - & - & - \\
\hline Otostegia tomentosa & 22.22 & 0.78 & 33.33 & 1.55 & - & - & - \\
\hline Pavetta abyssinica & 11.11 & 0.39 & 11.11 & 0.52 & - & - & - \\
\hline Phytolacca dodecandra & 5.56 & 0.19 & 11.11 & 0.52 & - & - & - \\
\hline Pluchea dioscoridis & 8.33 & 0.29 & 11.11 & 0.52 & - & - & - \\
\hline Premna schimperi & 55.56 & 1.95 & 55.56 & 2.59 & 0.21 & 0.43 & 4.97 \\
\hline Pterolobium stellatum & 127.78 & 4.48 & 77.78 & 3.63 & - & - & - \\
\hline Rhoicissus tridentata & 13.89 & 0.49 & 22.22 & 1.04 & - & - & - \\
\hline Rhus glutinosa & 16.67 & 0.58 & 33.33 & 1.55 & 0.20 & 0.41 & 2.54 \\
\hline Rhus vulgaris & 41.67 & 1.46 & 33.33 & 1.55 & 0.05 & 0.10 & 3.11 \\
\hline Ricinus communis & 8.33 & 0.29 & 11.11 & 0.52 & - & - & - \\
\hline Sapium ellipticum & 8.33 & 0.29 & 33.33 & 1.55 & 3.34 & 6.75 & 8.59 \\
\hline Schrebera alata & 22.22 & 0.78 & 33.33 & 1.55 & 0.08 & 0.15 & 2.48 \\
\hline Senna didymobotrya & 47.22 & 1.66 & 22.22 & 1.04 & - & - & - \\
\hline Senna singueana & 8.33 & 0.29 & 22.22 & 1.04 & - & - & - \\
\hline Sesbania sesban & 36.11 & 1.27 & 22.22 & 1.04 & - & - & - \\
\hline Solanum anguivi & 2.78 & 0.10 & 11.11 & 0.52 & - & - & - \\
\hline Steganotaenia araliacea & 25.00 & 0.88 & 33.33 & 1.55 & 0.02 & 0.03 & 2.46 \\
\hline Stereospermum kunthianum & 2.78 & 0.10 & 11.11 & 0.52 & - & - & - \\
\hline Syzygium guineense & 8.33 & 0.29 & 11.11 & 0.52 & 2.40 & 4.85 & 5.66 \\
\hline Triumfetta pilosa & 8.33 & 0.29 & 11.11 & 0.52 & - & - & - \\
\hline Vernonia myriantha & 55.56 & 1.95 & 33.33 & 1.55 & 0.10 & 0.19 & 3.69 \\
\hline Ximenia americana & 11.11 & 0.39 & 11.11 & 0.52 & - & - & - \\
\hline Total & $2,850.0$ & 100.00 & $2,144.44$ & 100.00 & 49.45 & 100.00 & 300.00 \\
\hline
\end{tabular}

